Louisiana State University

LSU Digital Commons

6-22-2009

\title{
Spatial and spectral properties of the high-order harmonic emission in argon for seeding applications
}

Xinkui He

Lunds Universitet

M. Miranda

Lunds Universitet

J. Schwenke

Lunds Universitet

O. Guilbaud

Lunds Universitet

T. Ruchon

Lunds Universitet

See next page for additional authors

Follow this and additional works at: https://digitalcommons.Isu.edu/physics_astronomy_pubs

\section{Recommended Citation}

He, X., Miranda, M., Schwenke, J., Guilbaud, O., Ruchon, T., Heyl, C., Georgadiou, E., Rakowski, R., Persson, A., Gaarde, M., \& L'Huillier, A. (2009). Spatial and spectral properties of the high-order harmonic emission in argon for seeding applications. Physical Review A - Atomic, Molecular, and Optical Physics, 79 (6) https://doi.org/10.1103/PhysRevA.79.063829

This Article is brought to you for free and open access by the Department of Physics \& Astronomy at LSU Digital Commons. It has been accepted for inclusion in Faculty Publications by an authorized administrator of LSU Digital Commons. For more information, please contact ir@lsu.edu. 


\section{Authors}

Xinkui He, M. Miranda, J. Schwenke, O. Guilbaud, T. Ruchon, C. Heyl, E. Georgadiou, R. Rakowski, A.

Persson, M. B. Gaarde, and A. L'Huillier 


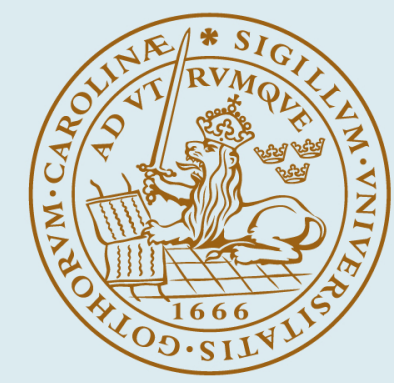

\section{LUND UNIVERSITY}

\section{Spatial and spectral properties of the high-order harmonic emission in argon for seeding applications}

He, Xinkui; Miranda, Miguel; Schwenke, Jörg; Guilbaud, Olivier; Ruchon, Thierry; Heyl, C.; Georgiadou, Elisavet; Rakowski, Rafal; Persson, Anders; Gaarde, Mette; L'Huillier, Anne

Published in:

Physical Review A (Atomic, Molecular and Optical Physics)

DOI:

10.1103/PhysRevA.79.063829

2009

Link to publication

Citation for published version (APA):

He, X., Miranda, M., Schwenke, J., Guilbaud, O., Ruchon, T., Heyl, C., Georgiadou, E., Rakowski, R., Persson, A., Gaarde, M., \& L'Huillier, A. (2009). Spatial and spectral properties of the high-order harmonic emission in argon for seeding applications. Physical Review A (Atomic, Molecular and Optical Physics), 79(6). https://doi.org/10.1103/PhysRevA.79.063829

Total number of authors:

11

\footnotetext{
General rights

Unless other specific re-use rights are stated the following general rights apply:

Copyright and moral rights for the publications made accessible in the public portal are retained by the authors and/or other copyright owners and it is a condition of accessing publications that users recognise and abide by the legal requirements associated with these rights.

- Users may download and print one copy of any publication from the public portal for the purpose of private study or research.

- You may not further distribute the material or use it for any profit-making activity or commercial gain

- You may freely distribute the URL identifying the publication in the public portal

Read more about Creative commons licenses: https://creativecommons.org/licenses/

Take down policy

If you believe that this document breaches copyright please contact us providing details, and we will remove access to the work immediately and investigate your claim.
} 


\title{
Spatial and spectral properties of the high-order harmonic emission in argon for
} seeding applications

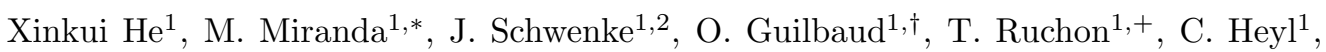 \\ E. Georgiadou ${ }^{1}$, R. Rakowski ${ }^{1}$, A. Persson ${ }^{1}$, M. B. Gaarde ${ }^{3}$ and A. L'Huillier ${ }^{1}$ \\ ${ }^{1}$ Department of Physics, Lund University, P. O. Box 118, SE-221 00 Lund, Sweden \\ ${ }^{2}$ Max-lab, Lund University, P. O. Box 118, SE-221 00 Lund, Sweden and \\ ${ }^{3}$ Department of Physics and Astronomy, Louisiana State University, Baton Rouge, LA 70803-4001
}

\begin{abstract}
We characterize and control the harmonic emission in the spectral and spatial domain in order to define in which conditions the harmonic radiation can be a high-quality seed for soft X-ray and $\mathrm{X}$-ray free electron lasers. The length of the gas cell where harmonics are generated was optimized and the energy per pulse was determined in absolute value with a calibrated X-ray photodiode. The beam spatial profile was measured and in some conditions a very collimated beam with a half angle divergence below one mrad could be obtained. We also show that increasing the intensity of the fundamental laser field leads to a considerable broadening of the bandwidth of the harmonic radiation, allowing us to cover a large spectral range. This effect is due to fundamental reshaping leading to efficient phase matching of both short and long trajectory contributions.
\end{abstract}

PACS numbers: 32.80.Rm, 32.80.Qk, 42.65.Ky

\section{INTRODUCTION}

A lot of effort is devoted world-wide to the development of coherent light sources in the extreme ultraviolet (XUV) range with laser-like properties. Different paths are being explored, from X-ray Free Electron Lasers (XFELs), based on the self amplification of the synchrotron radiation emitted by relativistic electron bunches [1] to soft X-ray Lasers (SXRLs), relying on the realization of a population inversion in highly charged ions obtained in a hot dense plasma [2]. These two approaches lead to high-energy XUV pulses with, in general, poor coherence properties compared to conventional lasers, since the XUV beams result from the direct amplification of the spontaneous emission emitted at one extremity of the medium. High-order harmonics emitted during the nonlinear interaction between an intense ultrashort laser and a gas [3, 4] inherit most of the desirable properties of the driving laser in terms of spatial and temporal coherence, but suffer from low conversion efficiencies, resulting in pulse energies typically in the $\mathrm{nJ}$ range [5]. A straightforward idea, explored in several laboratories around the world [6-8], is to use harmonic radiation to seed the first mentioned sources, thus combining the coherence and flexibility of the harmonics with the high output energy of X-FELs or SXRLs.

Seeding requires well-characterized and optimized harmonics. The spatial wavefront and spectral content should be of high quality and the energy per unit of bandwidth as high as possible to overcome the spontaneous emission in the amplifier. Many studies have been devoted to characterize the harmonic spectra $[9,10]$ or the spatial profile $[11,12]$ of high-order harmonics, however, often separately. In addition, the harmonic frequency should be matched to a given X-ray plasma spectral line. Previous work uses a high-intensity frequency-chirped fundamental field to spectrally modulate and, to some extent, tune the high energy part of the high-order harmonic generation spectrum [13, 14]. An adaptive spectral filter (DAZZLER) has also been used to provide some tunability of the high-harmonic spectrum [15].

In the present paper, we discuss the characteristics of the harmonic radiation generated in argon around $30 \mathrm{~nm}$ by a rather high energy laser driver with about $100 \mathrm{~mJ}$ per pulse in $40 \mathrm{fs}$ at $800 \mathrm{~nm}$ wavelength. The spectrum, spatial profile and energy per pulse are determined and optimized for different focusing geometries and medium lengths. We also show that the spectral bandwidth is considerably increased by simply using a higher laser intensity. We concentrate on the wavelength range between 20 and $40 \mathrm{~nm}$, which can be reached using Ar as generating gas. In Section 2, we describe the main physics of harmonic generation useful for this work. In Section 3, we present the experimental method and results.

\section{BRIEF SUMMARY OF THE PHYSICS OF HIGH-ORDER HARMONIC GENERATION}

\section{A. Single atom response}

The physical origin of harmonic generation can be easily understood by a simple semiclassical picture [16, 17]. When an atom is exposed to an intense infrared laser field, the atomic potential is considerably distorted by the strong electric field. An electron from the outer shell may tunnel through the Coulomb barrier and ionize. This electron is then accelerated by the laser field, driven back to the parent ion when the direction of the electric field changes sign, and may recombine to the ground state, thereby emitting a high energy photon. This energy is equal to the atomic ionization energy $\left(I_{p}\right)$ plus the energy acquired during the acceleration. This process occurs every half-cycle of the driving laser, so that the emitted ra- 
diation is periodic with a periodicity equal to $1.3 \mathrm{fs}$, for a $800 \mathrm{~nm}$ fundamental laser field. The spectrum consists of harmonic peaks at odd multiples of the incident frequency. The kinetic energy $\left(E_{c}\right)$ acquired during the electron excursion in the continuum can be calculated using a simple classical calculation, which provides to the experimentalist a useful guide to estimate the available spectral range $(W)$ according to the formula: $W=I_{p}+E_{c}$. Results of such calculations are presented in Fig. 1 (a). The kinetic energy gained is plotted in units of the ponderomotive energy $U_{p}$ related to the laser field amplitude $(E)$ by $U_{p}=e^{2} E^{2} / 4 m \omega^{2}$ where $e$ and $m$ are the charge and mass of the electron and $\omega$ the laser frequency. $U_{p}$ (expressed in eV) can also been written as $9.34 \lambda^{2} I$ where the laser wavelength $\lambda$ is in $\mu \mathrm{m}$ and the laser intensity $I$ in $10^{14} \mathrm{~W} / \mathrm{cm}^{2}$ units. For example, for $\lambda=0.8 \mu \mathrm{m}$, $I=2 \times 10^{14} \mathrm{~W} / \mathrm{cm}^{2}$, the maximum kinetic energy is 38 $\mathrm{eV}$ and the maximum photon energy generated in $\mathrm{Ar}$ is about $54 \mathrm{eV}$. The abscissa in Fig. 1(a) indicates the return time of the electron in units of the laser cycle $(2.7$ fs). This figure shows that there are two possible electron trajectories leading to the same return energy, below the maximum energy. The trajectory with the longer excursion time is called the long trajectory, while that with the shorter excursion time is called short.
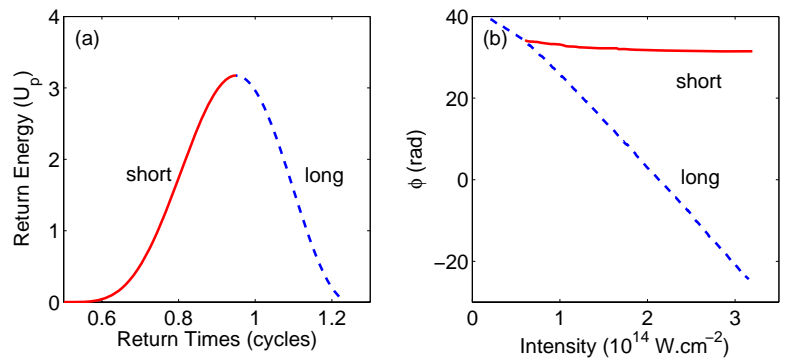

FIG. 1: (a) Classical calculation of the kinetic energy of the electron as a function of the return time. (b) Calculation of the intensity dependence of the $19^{t h}$ harmonic phase for the short and long trajectories using the strong field approximation [19].

The phase accumulated by the electron on these trajectories is transfered to the emitted radiation field. The phase of the harmonic light is therefore not simply related to the phase of the driving laser, but also includes an intrinsic phase component that can vary rapidly with laser intensity. This intrinsic phase which is weakly dependent on the process order, has consequences for both the spatial and spectral emission characteristics and we indicate in Fig. 1 its variation with intensity for the $19^{t h}$ harmonic generated in Ar, calculated using the Strong Field Approximation (SFA) [18, 19]. The two branches refer to the short (red) and long (blue) trajectories. For $I>10^{14} \mathrm{~W} . \mathrm{cm}^{-2}$, in the so-called plateau region, the intrinsic phase can be approximated by $\Phi_{j}(\mathbf{r}, t)=\alpha_{j} I(\mathbf{r}, t)$, where $j$ refers to the trajectory (short or long). The har-
TABLE I: Useful parameters for the $15^{\text {th }}$ to the $31^{\text {st }}$ harmonics in Ar. The intensity of the laser field used for the calculation is $1.5 \times 10^{14} \mathrm{~W} / \mathrm{cm}^{2}$, and the unit for $\alpha$ is $10^{-14} \mathrm{~W}^{-1} \mathrm{~cm}^{2}$.

\begin{tabular}{lrrrrrrr}
\hline Order & 19 & 21 & 23 & 25 & 27 & 29 & 31 \\
\hline$\lambda_{h}(\mathrm{~nm})$ & 42.1 & 38.1 & 34.8 & 32.0 & 29.6 & 27.6 & 25.8 \\
$\alpha_{s}$ & -1.0 & -1.8 & -2.7 & -3.8 & -5.1 & -6.9 & -9.8 \\
$\alpha_{l}$ & -22.9 & -22.2 & -21.5 & -20.5 & -19.2 & -17.5 & -14.8 \\
$\theta_{s}(\mathrm{mrad})$ & 0.3 & 0.4 & 0.6 & 0.8 & 1.0 & 1.2 & 1.6 \\
$\theta_{l}(\mathrm{mrad})$ & 6.1 & 5.4 & 4.8 & 4.2 & 3.6 & 3.1 & 2.4 \\
$\Delta \lambda_{s}(\mathrm{~nm})$ & 0.2 & 0.2 & 0.2 & 0.3 & 0.4 & 0.5 & 0.5 \\
$\Delta \lambda_{l}(\mathrm{~nm})$ & 3.2 & 2.6 & 2.1 & 1.7 & 1.4 & 1.0 & 0.8 \\
\hline
\end{tabular}

monic field is the coherent sum of two contributions:

$$
E_{h}(\mathbf{r}, t)=\sum_{s, l} A_{h j}(\mathbf{r}, t) e^{i \omega_{h} t-i \alpha_{j} I(\mathbf{r}, t)}
$$

where $A_{h j}$ denotes the amplitude of the contribution of the trajectory $j$ to the harmonic field with frequency $\omega_{h}$. We also indicate in Table 1 the values of these $\alpha$ coefficients for the short and long trajectories, for a few harmonics discussed in the present work [19]. Assuming a Gaussian distribution for the fundamental and harmonic fields, both in space and time, we can estimate the divergence and spectral bandwidth of the harmonic field according to:

$$
\theta_{j}=\frac{\lambda_{h}}{\pi w_{h}} \sqrt{1+4 \alpha_{j}^{2} I_{0}^{2} \frac{w_{h}^{4}}{w_{f}^{4}}}
$$

and:

$$
\Delta \lambda_{j}=\frac{\lambda_{h}^{2}}{\pi c \tau_{h}} \sqrt{1+4 \alpha_{j}^{2} I_{0}^{2} \frac{\tau_{h}^{4}}{\tau_{f}^{4}}}
$$

$\lambda_{h}, w_{h}, \tau_{h}$ denote the wavelength, beam waist and pulse width of the harmonic field and $w_{f}, \tau_{f}, I_{0}$ the beam waist, pulse width and peak intensity of the fundamental field. If the second terms in the roots are negligible, the harmonic field is Fourier transform limited in the time domain, and diffraction limited in space. In general, phase effects lead to deviation from the Fourier limit, especially for high intensities and long trajectories. Upper values for $\theta_{j}(j=s, l)$ and $\Delta \lambda_{j}$ are given in table 1 using $w_{f}=150 \mu \mathrm{m}, \tau_{f}=40 \mathrm{fs}$ and $I_{0}=1.5 \times 10^{14}$ $\mathrm{W} / \mathrm{cm}^{2}$, representing typical experimental values and assuming $w_{h} / w_{f}=\tau_{h} / \tau_{f}=1$. In reality these ratios are slightly below one, and decrease with process order, since high-order harmonics require higher laser intensity to be generated and are emitted over a smaller diameter and shorter pulse duration. The values indicated in the table might be used as a guide to estimate upper values for the spatial and spectral widths of the HHG radiation. 


\section{B. Propagation}

The optimization of the harmonic emission requires not only a strong single atom response but also that all of the atoms in the medium emit in phase. For the sake of simplicity, we here give a simple description of propagation using a one-dimension approximation, along the propagation axis $(z)$. More advanced calculations accounting also for off-axis effects will be presented in Section III. Phase matching is realized when the variation of the difference between the phase of the $q^{t h}$ harmonic field generated in the medium and that of the polarization driving it, equal to

$$
\delta \Phi_{q}(z, t)=\int \Delta k_{q}\left(z^{\prime}, t\right) d z^{\prime}+q \arctan \left(z / z_{R}\right)+\alpha_{j} I(z, t),
$$

is minimized over the medium length [20]. In the right side of Eq. (4), the first term denotes the influence of dispersion, which includes two opposite contributions, from the neutral medium and the free electrons. The second term indicates the influence of focusing $\left(z_{R}\right.$ denoting the Rayleigh length of the fundamental beam). Finally, the third term is the single-atom phase described above, which strongly depends on the trajectory. For loose focusing geometries, in general, the contribution of dispersion effects is stronger than the geometrical and singleatom phase variations, both for a neutral medium and for a strongly ionized medium. The first condition to achieve phase matching is to (approximately) cancel the neutral atom and free electron dispersion, which is achieved for a degree of ionization of the medium of approximately 7 $\%$ in argon. The phase variation due to the geometrical phase and to the single-atom phase (especially in the case of the long trajectory) needs to be included for a correct description of phase matching $[21,22]$. In addition, absorption in general limits the conversion efficiency [23].

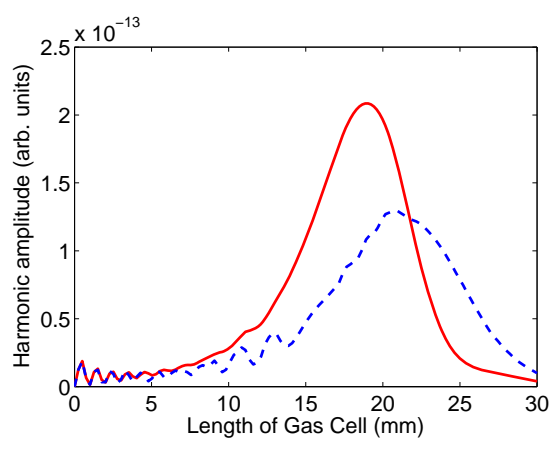

FIG. 2: Phase matching contribution to the harmonic intensity as a function of cell length for short (red solid) and long (blue dashed) trajectories.

Figure 2 shows results of calculations performed for the $21^{\text {st }}$ harmonic in argon using a one-dimension model described in [24], including all of the phase terms in Eq. (4), and consisting in summing all of the microscopic contributions over a certain length. The red and blue curves re- fer to the contribution of the short and long trajectories, considered separately in the calculation, with the same single atom response amplitude. The oscillations observed for short length media indicate that phase matching is not realized and that the field generated at some location in the medium is cancelled by that generated in another location. The quadratic increase from $L=12 \mathrm{~mm}$ is characteristic of phase matching. The harmonic yield saturates and eventually decreases when the absorption limit is reached. The phase variation for the long trajectory contribution leads to a shift in the optimal medium length. The curves shown in Fig. 2 depend only weakly on the process order: for example, the short trajectory contribution of the $15^{\text {th }}$ (resp. $25^{\text {th }}$ ) harmonic is maximized for $L=16 \mathrm{~mm}$ (resp. $L=20 \mathrm{~mm}$ ). These results obtained with a one-dimensional model might change a little when generalizing to three dimensions, when considering other laser intensities and gas pressures. Our aim here was to illustrate the physics of phase matching of high-order harmonics rather than simulate a realistic experimental situation.

The conclusion of our model is that efficient, absorption-limited, phase matching of high-order harmonics may be achieved by using sufficiently long media. Another way would be to have a cell with a length corresponding to the absorption length (or equal to a few times the absorption length) and to locate it at the position where the intensity is such that phase matching can be achieved. Experimentally, the first solution is much easier. Other effects such as a spatio-temporal modification of the fundamental field [25], leading in particular to defocusing, could also favor phase matching of highorder harmonic generation in long media [26] (see also the discussion below).

\section{EXPERIMENTAL METHOD AND RESULTS}

A. Measurement of spatial and spectral profiles.

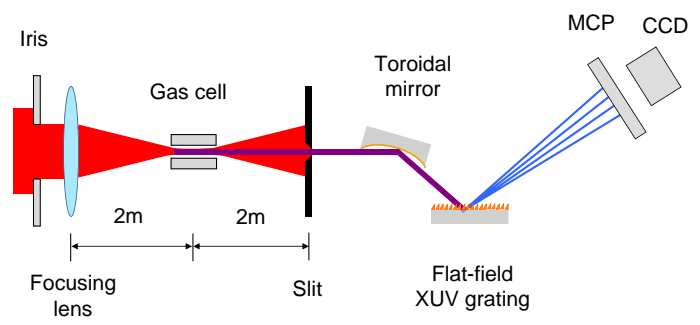

FIG. 3: Experimental setup.

Our experimental setup is shown in Fig. 3. The laser is an amplified Ti:Sapphire $10 \mathrm{~Hz}$ system delivering 40 fs pulses around $800 \mathrm{~nm}$ with an energy up to $1 \mathrm{~J}$. The results presented below are obtained with only a frac- 
tion of this energy, less than 100 mJ. Furthermore, the $50 \mathrm{~mm}$ diameter beam is apertured down by an iris with variable diameter (with a diameter typically between 11 and $16 \mathrm{~mm}$ ), so that only about a few mJ infrared energy is actually sent into our experimental setup. The beam is focused by a lens with $2 \mathrm{~m}$ focal length into a gas cell with $1 \mathrm{~mm}$ transverse diameter and variable length (from 3 to $20 \mathrm{~mm}$ ). The harmonic spectra are detected by a flat-field XUV spectrometer, with a $100 \mu \mathrm{m}$ entrance slit, located at approximately $2 \mathrm{~m}$ from the gas jet. An XUV micro channel plate (MCP) combined with a CCD camera are used to capture the final image. In contrast to many previous measurements $[11,12]$, we do not image the harmonic source, but a slice of its profile at a relatively long distance $(2 \mathrm{~m})$ from the source, which gives us the possibility to measure the spatial profile in the far field (and therefore the divergence) of the beam [27, 28]. Our spectral resolution is estimated to be $0.2 \mathrm{~nm}$.

A typical experimental spectrum is shown in Fig. 4. Harmonics are shown from the $19^{\text {th }}$ to the $33^{\text {rd }}$, corresponding to a spectral range of 42 to $24 \mathrm{~nm}$, or in energy, 29 to $51 \mathrm{eV}$. Spatial profiles are shown in the horizontal direction, while the vertical one shows spectra. Fig. 4 (b) shows some spatial profiles, while the spatially-integrated spectrum is presented in (c). These measurements show evidence for contributions from the short trajectory, leading to a narrow collimated beam and also from the (weaker) long trajectory, with much higher divergence. In the cutoff region, both contributions merge together.

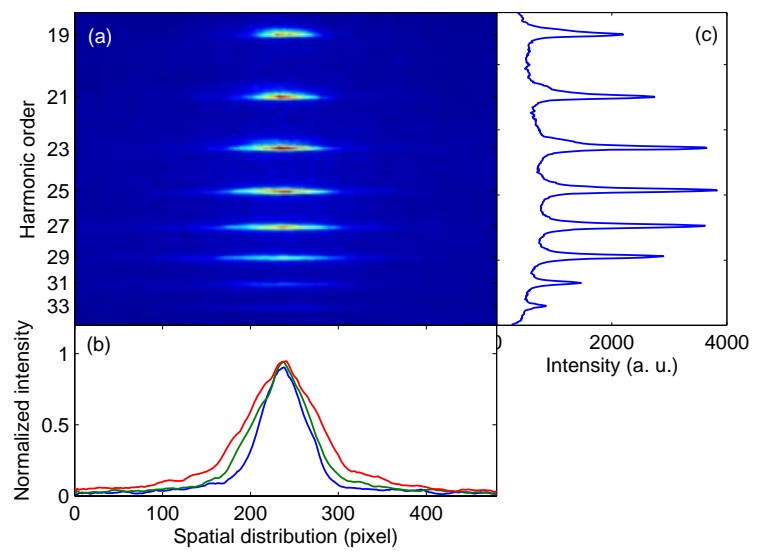

FIG. 4: (a) Experimental measurement using a $21 \mathrm{~mm} \mathrm{Ar}$ gas cell and a fundamental intensity of $I=10^{14} \mathrm{~W} / \mathrm{cm}^{2}$; (b) Spatial profile of the $19^{\text {th }}$ (blue), $23^{\text {rd }}$ (green) and $27^{\text {th }}$ (red) harmonic (c) Spatially integrated spectrum.

In order to avoid any influence of the spectrometer collecting optics on the harmonic spatial profile, we have measured it directly by taking an image with the MCP at about $2 \mathrm{~m}$ from the gas cell. The fundamental field and the low-order harmonics (up to the $11^{\text {th }}$ ) were removed by absorption in a $200 \mathrm{~nm}$-thin Al filter. A typical result is shown in Fig. 5. A relatively narrow peak is super- posed on a broad pedestal, which can be attributed to the main two quantum paths responsible for harmonic generation. The harmonic beam diameter could be measured by comparing with the diameter of the aperture on which the filter was mounted, and which could be observed on the MCP. The full width at half maximum is $1.4 \mathrm{~mm}$ and the corresponding beam divergence is 0.7 mrad. This agrees with the predictions presented above (Table 1) for the (dominant) short trajectory and for the strong $23^{t h}-25^{\text {th }}$ harmonics. The ratio between the short and long trajectory contributions is affected by the position of the focus of the infrared beam in the gas cell. In agreement with previous work [21], we find that the short trajectory is enhanced compared to the long one when the focus position is before the gas cell.

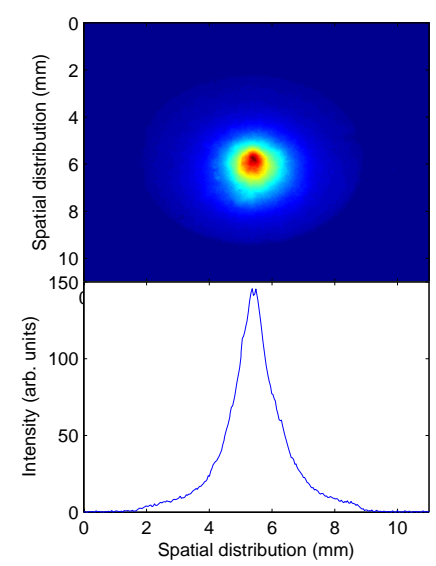

FIG. 5: Spatial profile of the total harmonic yield transmitted through the Al filter measured at $2 \mathrm{~m}$ from the $15 \mathrm{~mm}$ gas cell. The infrared beam was focused just at the beginning of the cell. The aperture was $14 \mathrm{~mm}$.

\section{B. Measurement of the harmonic pulse energy}

The pulse energy of the high-order harmonic radiation was measured by using a calibrated XUV photodiode, with good sensitivity from $1 \mathrm{eV}$ to $6 \mathrm{keV}$. The diode could be moved under vacuum before the flat-field XUV spectrometer, so that we could measure the energy and the corresponding spectrum within the same series of measurements. Two $200 \mathrm{~nm} \mathrm{Al} \mathrm{filters} \mathrm{were} \mathrm{needed} \mathrm{to} \mathrm{block}$ the fundamental beam. The number of the electrons $N_{e}$ generated from the photodiode is the integral of the measured current. The number of the photons at each harmonic frequency is obtained through the equation

$$
N_{e}=\sum_{q} \eta_{q} t_{q} N_{q}
$$

where $N_{q}$ denotes the number of photons generated at the $q^{t h}$ harmonic frequency, $t_{q}$ is the transmission by the $\mathrm{Al}$ filters and $\eta_{q}$ is the quantum efficiency of the photo- 
diode for the $q^{t h}$ harmonic. The absorption from the $\mathrm{Al}$ filters can be estimated by calculating the transmission function of $\mathrm{Al}$, accounting for the effect of a thin layer of oxide, which unfortunately reduces the transmission and introduces an uncertainty in the energy determination. Fig. 6 shows the transmission of $200 \mathrm{~nm} \mathrm{Al} \mathrm{(blue}$ line), as well as that of $200 \mathrm{~nm} \mathrm{Al}$, plus $20 \mathrm{~nm}$ oxide (10 nm on each side), which is an rough estimation of the oxide layer on the filters used in this experiment [29]. From the relative ratios between the generated harmonics, obtained from spectral measurements, the number of photons per harmonic, the pulse energy, as well as the conversion efficiency can be deduced. Results of measurements performed in optimized conditions (with a 19 mm length gas cell, see below) are presented in Table II.

TABLE II: Optimized high order harmonic energy and conversion efficiency.

\begin{tabular}{lrrrrrr}
\hline Harmonic order & 17 & 19 & 21 & 23 & 25 & 27 \\
\hline Transmission & 0.12 & 0.13 & 0.15 & 0.16 & 0.19 & 0.21 \\
Photon number $\left(\times 10^{10}\right)$ & 3.03 & 2.98 & 3.10 & 2.63 & 1.60 & 1.41 \\
Energy $(\mu \mathrm{J})$ & 0.13 & 0.14 & 0.16 & 0.15 & 0.10 & 0.09 \\
Efficiency $\left(\times 10^{-6}\right)$ & 6.8 & 7.5 & 8.6 & 8.0 & 5.3 & 5.0 \\
\hline
\end{tabular}

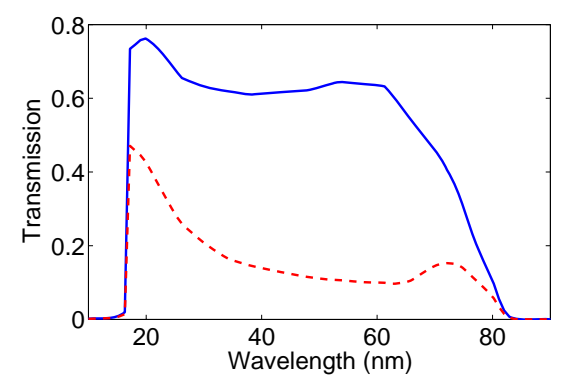

FIG. 6: Calculated transmission function for $200 \mathrm{~nm} \mathrm{Al} \mathrm{filter}$ (blue solid) and with $10 \mathrm{~nm}$ oxidation layer on each side (red dashed).

\section{Optimisation of the output energy by varying the cell length.}

Figure 7 (a) shows the variation of the harmonic energy as a function of cell length for a few harmonics. The focus was located at the center of the cell. The saturation effect observed is attributed to absorption, which is more important for the low order harmonics than for the high order ones. Fig 7 (b) shows the $23^{\text {rd }}$ harmonic spatial profile obtained at $\sim 2 \times 10^{14} \mathrm{~W} / \mathrm{cm}^{2}$, with gas cells of different lengths and an aperture before the lens equal to $13 \mathrm{~mm}$. The central part of the angular profile, which reflects the contribution from the short trajectory, is approximately constant with order and with cell length, of the order of $1 \mathrm{mrad}$.

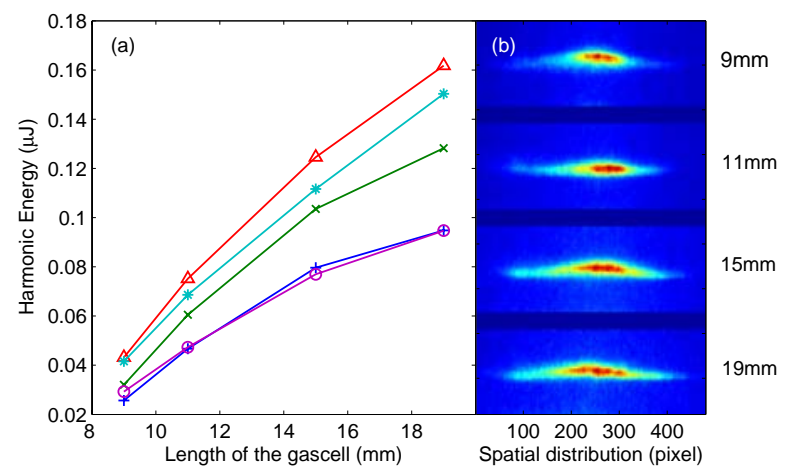

FIG. 7: (a) Harmonic energy as a function of cell length for the $13^{\text {th }}$ (line with plus), $17^{\text {th }}$ (line with $\mathrm{x}$-mark), $21^{\text {st }}$ (line with triangle), $23^{\text {rd }}$ (line with star) and $27^{\text {th }}$ (line with circle) order. (b) Spatial distribution of $23^{\text {rd }}$ harmonic for different gas cell lengths.

Increasing the cell length results in an increased harmonic energy as well as the appearance of the long trajectory contribution, with higher divergence. The predictions of our model (see Fig. 2) agree quite well with the experimental observation, showing a saturation for medium lengths of $20 \mathrm{~mm}$ and an increase of the contribution of the long trajectory as the length increases. The latter effect is of interest for seeding applications, since the contribution of the long trajectory can be made spectrally broader at high laser intensity, thus providing increased possibility to match the gain profile of an amplifier, as explained below.

\section{Increasing the spectral range between consecutive harmonics}

Spectra obtained from two different fundamental energies by changing iris diameters are shown in Fig. 8. The harmonic spectra get broadened as the input energy increases, more than the prediction of Eq. (3) for a single trajectory. In addition, interference structures appear. This complicated structure appears in connection with an ionization-induced spatio-temporal reshaping of the laser beam, which improves the phase matching conditions for the long trajectory. The short trajectory is well phase matched both before and after the reshaping, and we therefore see spectral interference between the contributions from both trajectories [30]. Similar structures have been observed in previous work [27, 28] and interpreted in terms of interference between the contribution of the two (short and long) trajectories, without, however, explaining the reason for both contributions to be of the same strength.

Our interpretation is supported by theoretical calculations including both the microscopic and macroscopic response of the argon gas to the intense laser pulse. The re- 


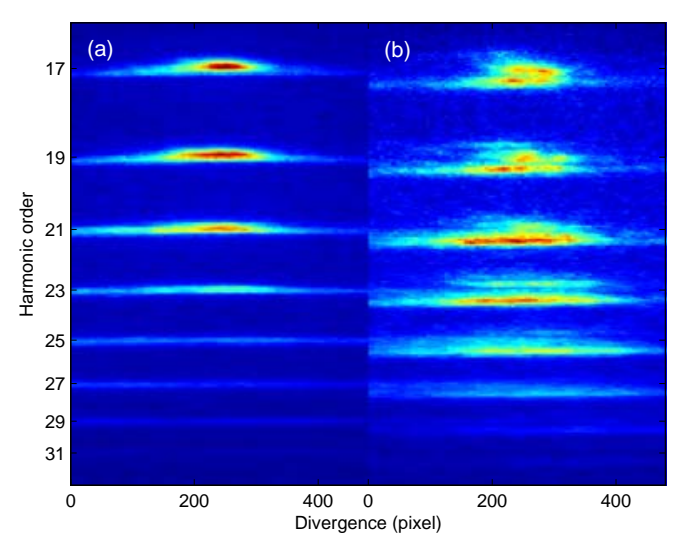

FIG. 8: Harmonic spectra at two different intensities, measured to be $2 \times 10^{14} \mathrm{~W} / \mathrm{cm}^{2}$ (a) and $\sim 5 \times 10^{14} \mathrm{~W} / \mathrm{cm}^{2}$ (b). A $21 \mathrm{~mm}$ gas cell was used and the focus point of the infrared beam was in the middle of the cell. Note the significant blue shift of the spectral distribution at high intensity.

sults were calculated via the coupled, non-adiabatic solutions of the time-dependent Schrödinger equation, within the strong field approximation [17], and the Maxwell wave equation. Our approach is described in detail in [20]. As initial conditions for the calculation we use similar parameters as the experiment, in terms of peak intensity, duration, and focusing conditions for the laser beam, and density and length of the argon cell.

The calculated far field spatio-spectral profiles of harmonics 17 through 21 are shown in Fig. 9(a) and (b). At low intensity the very loose focusing conditions strongly favors phase matching of the short trajectory, and the resulting harmonics are spectrally narrow and well behaved. The contribution from the long trajectory in these conditions can barely be seen in Fig. 9(a) as a faint halo around the $19^{\text {th }}$ harmonic, most prominent on the lowfrequency side and at divergences above $0.6 \mathrm{mrad}$. At high intensity, the harmonic spectra are much broader, exhibit interference fringes, and are blue-shifted relative to the low-intensity case. As mentioned above, the interference is the result of both short and long trajectory contributions being well phase-matched by the strongly reshaped laser beam. Fig. 9(c) and (d) show the spatiotemporal profile of the laser beam in the near field, at the end of the argon medium. The high-intensity beam has undergone violent spatio-temporal reshaping which results in defocusing, strong blue-shifting on axis, and the overall change in shape where the laser envelope reaches its maximum at different times for different radii. The reshaping happens after a few $\mathrm{mm}$ of propagation and then does not change much through the remainder of the medium. In the calculations, we see a strong increase in the long-trajectory contribution around the propagation distances where the reshaping sets in, with a significant component on axis. The short and long trajectory contributions thus interfere in the far field, leading to the two or three horizontal stripes observed in Fig. 9(b) and $8(\mathrm{~b})$.

This effect provides an easy way for covering a larger spectral range, which is very important for seeding $\mathrm{X}$ ray laser plasmas. For example, the $25^{\text {th }}$ harmonic (32 $\mathrm{nm}$ ) is close to the X-ray lasing lines of $31.2 \mathrm{~nm}$ (Ne-like $\mathrm{Sc}$ ), $32.6 \mathrm{~nm}$ (Ne-like Ti), and $32.8 \mathrm{~nm}$ (Ni-like $\mathrm{Kr}$ ) and can be broadened to reach these lines, simply by increasing the laser intensity. It has been suggested previously $[13,14]$ that harmonics could be tuned by changing the chirp of the fundamental field. In such an experiment, several parameters are varied at the same time: chirp, pulse duration and laser intensity. We believe that the main effect is due to the variation of the laser intensity. ln addition, the fundamental chirp may induce additional or reduced spectral broadening depending on the sign of the chirp.
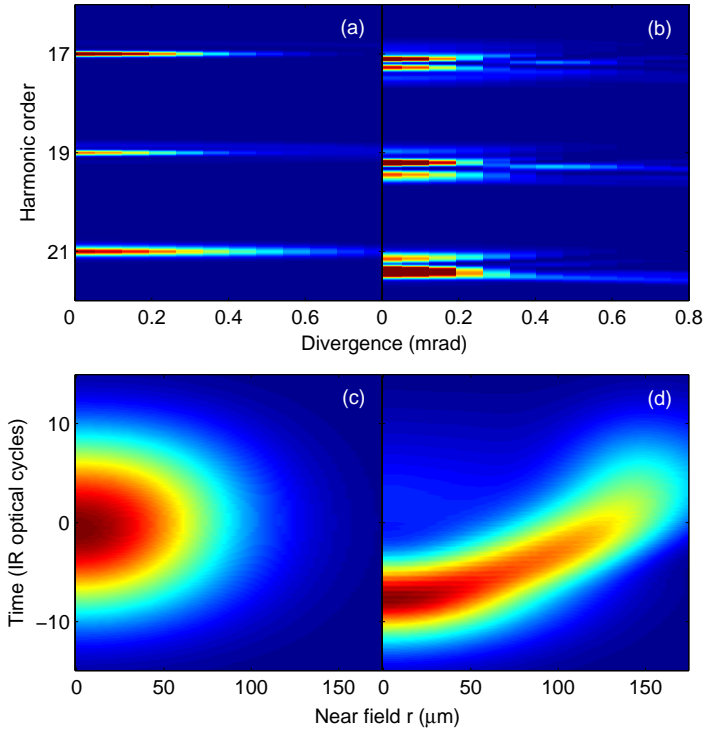

FIG. 9: Caculated far field spatial profile of different harmonic for two different peak intensities, $1 \times 10^{14} \mathrm{~W} / \mathrm{cm}^{2}$ (a) and $3.3 \times 10^{14} \mathrm{~W} / \mathrm{cm}^{2}$ (b). Spatio-temporal distribution of corresponding fundamental beam at the exit of the medium (c) and (d).

\section{CONCLUSION}

In this work we have studied the spectral and spatial properties of high-order harmonics generated in argon gas. The absolute value of the energy emitted per harmonic pulse was estimated in optimized conditions. We found that the use of long gas cells $(20 \mathrm{~mm})$ led to higher energies and more collimated XUV beams. Large spectral bandwidths, close to the interval between consecutive harmonics, could be achieved by increasing the intensity of the infrared beam in the gas cell leading to improved phase matching of the long trajectory. 


\section{Acknowledgments}

This research was supported by the European project NEST-ADVENTURE 012843-2 TUIXS, the Marie Curie actions EST MAXLAS, IIF OHIO and EIF Attotech, the Knut and Alice Wallenberg Foundation, the Swedish Science Council and the U. S. National Science Foundation through Grant No. PHY-044923. We thank B. Schütte, T. Eberle, J. Klemmer, F. Geier, E. Pourtal, for their help in some early stage of this experiment.

Present addresses:

*IFIMUP/IN, Department of Physics, University of Porto, Portugal

${ }^{\dagger}$ LIXAM, CNRS,Univ Paris-Sud, UMR n 8624, Orsay F-91405, France

+CEA-Saclay, DSM, Service des Photons, Atomes et Molcules, 91191, Gif-sur-Yvette, France
[1] J. Feldhaus, J. Arthur, and J. B. Hastings, J. Phys. B: At. Mol. Opt. Phys. 38, S799 (2005).

[2] D. L. Matthews et al., Phys. Rev. Lett. 54, 110 (1985).

[3] A. McPherson et al., J. Opt. Soc. Am. B 4, 595 (1987).

[4] M. Ferray et al., J. Phys. B 21, L31 (1988).

[5] Harmonic energies per pulse of the order of a $\mu \mathrm{J}$ have been reached by several groups, see, for example, J.-F. Hergott et al., Phys. Rev. 66, 021801 (2002) and E. Takahashi et al. J. Opt. Soc. Am. 20, 1, 158 (2003).

[6] P. Zeitoun et al., Nature 431, 426 (2006).

[7] Y. Wang et al., Nature Photonics 2, 94, (2008).

[8] G. Lambert et al., Nature Physics 4, 296 (2008).

[9] L. Nugent-Glandorf et al., Phys. Rev. A 62, 023812 (2000).

[10] H. T. Kim et al., J. Phys. B 37, 1141 (2004).

[11] J. W. G. Tisch et al., Phys. Rev. A 49, R28 (1994).

[12] P. Salières et al. J. Phys. B 27, L217 (1994).

[13] H. T. Kim et al., Phys. Rev. A 67, 051801(R) (2003).

[14] Z. Chang et al., Phys. Rev. A 58, R30 (1998).

[15] D. H. Reitze et al., Opt. Lett. 29, 86 (2004).
[16] P. B. Corkum, Phys. Rev. Lett. 71, 1994 (1993).

[17] M. Lewenstein et al., Phys. Rev. A 49, 2117 (1994).

[18] M. Lewenstein et al., Phys. Rev. A 52, 4747 (1995).

[19] K. Varju et al., Journal of Modern Optics 52, 379 (2005).

[20] M. B. Gaarde, J. L. Tate and K. J. Schafer, J. Phys. B 41132001 (2008).

[21] P. Salières, A. L'Huillier and M. Lewenstein, Phys. Rev. Lett. 743776 (1995).

[22] F. Lindner et al., Phys. Rev. A 68, 013814 (2003).

[23] E. Constant et al. Phys. Rev. Lett. 821668 (1999).

[24] T. Ruchon et al., New J. Phys. 10, 025027 (2008)

[25] M. B. Gaarde, M. Murakami and R. Kienberger, Phys. Rev. A 74, 053401 (2006).

[26] Y. Tamaki et al., Phys. Rev. Lett. 82, 1424 (1999)

[27] E. Brunetti et al. Phys. Rev. A 77, 023422 (2008).

[28] Han Xu et al., Phys. Rev. A 78, 033841 (2008).

[29] K. Varjú et al., Laser Physics 15, 888 (2005).

[30] A. Zair et al., Phys. Rev. Lett. 100, 143902 (2008). 